\title{
Epac2a-knockout mice are resistant to dexamethasone-induced skeletal muscle atrophy and short-term cold stress
}

\author{
Seung-Eun Song ${ }^{1, \#}$, Su-Kyung Shin ${ }^{1, \#}$, So-Young Park ${ }^{2}$, Il-Seon Hwang ${ }^{1}$, Seung-Soon Im ${ }^{1}$, Jae-Hoon Bae ${ }^{1}$, \\ Myung-Sook Choi ${ }^{3}$ \& Dae-Kyu Song ${ }^{1, *}$ \\ ${ }^{1}$ Department of Physiology \& Obesity-mediated Disease Research Center, Keimyung University School of Medicine, Daegu 82601, \\ ${ }^{2}$ Department of Physiology, Yeungnam University School of Medicine, Daegu 42415 , ${ }^{3}$ Department of Food Science and Nutrition, \\ Kyungpook National University, Daegu 41566, Korea
}

\begin{abstract}
Exchange protein directly activated by cAMP (Epac) 2a-knockout (KO) mice exhibit accelerated diet-induced obesity and are resistant to leptin-mediated adipostatic signaling from the hypothalamus to adipose tissue, with sustained food intake. However, the impact of Epac2a deficiency on hypothalamic regulation of sympathetic nervous activity (SNA) has not been elucidated. This study was performed to elucidate the response of Epac2a-KO mice to dexamethasone-induced muscle atrophy and acute cold stress. Compared to age-matched wild-type mice, Epac2a-KO mice showed higher energy expenditures and expression of myogenin and uncoupling protein-1 in skeletal muscle (SM) and brown adipose tissue (BAT), respectively. Epac2a-KO mice exhibited greater endurance to dexamethasone and cold stress. In wild-type mice, exogenous leptin mimicked the responses observed in Epac2a-KO mice. This suggests that leptin-mediated hypothalamic signaling toward SNA appears to be intact in these mice. Hence, the potentiated responses of SM and BAT may be due to their high plasma leptin levels. [BMB Reports 2018; 51(1): 39-44]
\end{abstract}

\section{INTRODUCTION}

Exchange protein directly activated by cAMP (Epac) is critical for cAMP signaling with cAMP-dependent protein kinases (1). Epac proteins are encoded by two distinct genes, yielding Epac1 and Epac2 (Rapgef4). Epac1 is widely expressed, whereas the three mRNA splice variants of Epac2 are differen-

${ }^{*}$ Corresponding author. Tel: +82-53-580-3873; Fax: +82-53-5803785; E-mail: dksong@kmu.ac.kr; dksong@dsmc.or.kr

${ }^{\text {\#} T h e s e ~ a u t h o r s ~ c o n t r i b u t e d ~ e q u a l l y ~ t o ~ t h i s ~ w o r k . ~}$

https://doi.org/10.5483/BMBRep.2018.51.1.132

Received 14 July 2017, Revised 7 August 2017,

Accepted 23 November 2017

Keywords: Brown adipose tissue, Epac2a, Leptin, Skeletal muscle, Sympathetic nervous activity tially expressed; Epac2a is mainly expressed in the brain, heart, and beta cells $(2,3)$. Epac2a-KO mice have normal body weight, glucose tolerance, and insulin sensitivity, at least until 12 weeks of age, but exhibit mild hyperleptinemia and have larger white adipocyte lipid droplets than those in agematched wild-type (WT) mice (4). The arcuate nucleus (ARH) and ventromedial hypothalamus, which largely determine the degree of body adiposity, express Epac2a, and Epac2a-deletion exhibited leptin resistance (4). This suggests that hypothalamic Epac2a is vital for appropriate leptin signaling to regulate food satiety.

Leptin is a critical hormone that links energy intake and storage to energy consumption (5). Non-obese humans and animals are responsive to exogenous leptin (6-8), which increases food satiety and energy consumption. Plasma leptin levels increase in proportion to adipose tissue mass to facilitate its role in the hypothalamus (9-11). The ARH responds to leptin and regulates the release of orexigenic and anorexigenic hormones (5). Appetite control by anorexigenic hormones is mediated by melanocortin 4 receptors (MC4R) (12). However, at the high leptin levels that are observed in the obese state, the hypothalamus becomes less responsive to exogenous leptin.

Hypothalamic leptin signaling elevates sympathetic nervous activity (SNA) to decrease body fat mass by increasing energy expenditure (EE), which is mediated by both MC4R-dependent and independent pathways (13-15). Obesity is associated with high blood pressure and hyperleptinemia, implying that leptin resistance does not influence the leptin-mediated hypothalamic activation of SNA (16). In addition, under leptin resistance, the effects of leptin on sympathetic tone in brown adipose tissue (BAT) were maintained by signaling from the dorsomedial hypothalamus (13).

The distribution of Epac2a in the distinct nuclei of the hypothalamus and its role in the leptin-mediated hypothalamic regulation of SNA has not been fully clarified. Thus, this study aimed to evaluate the possible changes in SNA in Epac2a-KO mice, by observing the response of peripheral tissues to dexamethasone-induced muscle atrophy and cold stress. Because the skeletal muscle and BAT possess plasmalemmal 

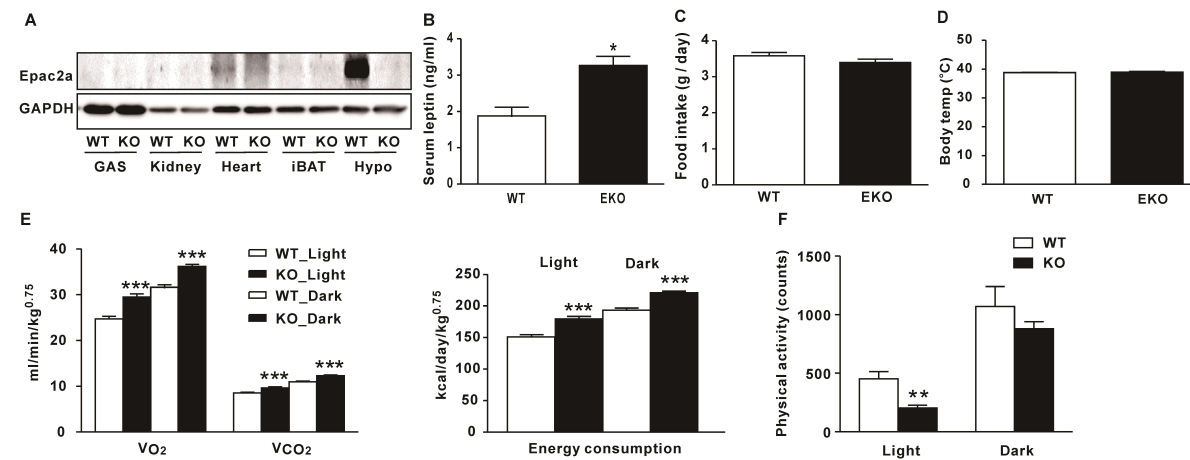

G
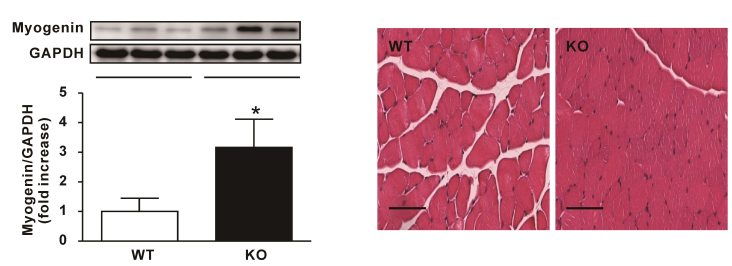

Fig. 1. Characteristics of Epac2a-KO mice. (A) Tissue distribution of Epac2a. Comparison of WT and Epac2a-KO mice by fasting serum leptin levels $(B, n=4)$, food intake $(C, n=5)$, rectal temperature $(\mathrm{D}, n=9)$, energy consumption $(\mathrm{E}, n=$ 6), and physical activity ( $\mathrm{F}, n=12)$. Myogenin mRNA $(n=15)$ and protein $(n$ $=3$ ) expression and cross sectional appearance $(\mathrm{G})$ of the gastrocnemius muscle in WT and $\mathrm{KO}$ mice. $\mathrm{H}$ \& $\mathrm{E}$ staining. Scale bars: $100 \mu \mathrm{m} .{ }^{*} \mathrm{P}<0.05$, $* * P<0.01$, and $* * * P<0.001$ compared to WT. Data represent the mean \pm SEM. GAS: gastrocnemius muscle; Hypo: hypothalamus.

long-form leptin receptors (OB-Rb) (17) as well as sympathetic G-protein-coupled receptors, the results may be helpful for understanding whether leptin signaling in myocytes and brown adipocytes is affected under hypothalamic leptin resistance and hyperleptinemia.

\section{RESULTS}

\section{Characteristics of Epac2a-KO mice}

Epac2a-KO mice appeared to be normal when compared to WT mice. We checked the protein expression of Epac2a in WT mice. Consistent with previous reports (3), the hypothalamus and heart expressed Epac2a but interscapular BAT (iBAT) and gastrocnemius muscle did not express Epac2a (Fig. 1A). Remarkably, Epac2a-KO mice had elevated fasting serum leptin levels (Fig. 1B), but food intake was not different from that of WT mice (Fig. 1C). No difference in rectal temperature was detected between the two groups (Fig. 1D). Oxygen consumption $\left(\mathrm{VO}_{2}\right)$, carbon dioxide production $\left(\mathrm{VCO}_{2}\right)$, and $\mathrm{EE}$ in $\mathrm{KO}$ mice were higher than those in WT mice (Fig. 1E), although the physical activity of $\mathrm{KO}$ mice was lower compared to WT mice (Fig. 1F). Myogenin levels were increased in the muscle of Epac2a-KO mice compared to the expression in $\mathrm{WT}$ mice (Fig. 1G). In addition, the cross-section area (CSA) of the muscle fibers was greater in $\mathrm{KO}$ mice. The average CSA of muscle fiber was $1546.6 \pm 56.04 \mu \mathrm{m}^{2}$ and $1808.9 \pm 75.50$ $\mu \mathrm{m}^{2}$ in WT and Epac2a-KO mice, respectively $(n=6)$.

Epac2a-KO mice show greater endurance to acute cold stress To observe the activity of BAT in Epac2a-KO mice, the mice were exposed to a cold environment $\left(4^{\circ} \mathrm{C}\right)$ for $6 \mathrm{~h}$. Epac2a-KO mice better-maintained body temperature in response to cold stress, and the decrease in body temperature induced by cold exposure was smaller than that in WT mice (Fig. 2A). The mass of iBAT and white adipose tissue at the thermoneutral condition was less in $\mathrm{KO}$ mice than in WT mice (Fig. 2B), consistent with the greater $\mathrm{EE}$ in $\mathrm{KO}$ mice. Nevertheless, the magnitude of cold stress-induced fat-mass decrease was similar between the two groups. Serum creatine kinase concentrations in the two groups were not different (data not shown), indicating that the shivering activity of the skeletal muscle did not differ. Comparison of the expression of thermogenesisrelated proteins in iBAT showed greater cold stress-mediated elevation in the expression of uncoupling protein-1 (UCP-1), peroxisome proliferator-activated receptor gamma coactivator 1 alpha (PGC-1 $\alpha)$, CCAAT-enhancer-binding proteins beta (C/EBP $\beta)$, carnitine palmitoyltransferase $1 \mathrm{~b}(\mathrm{CPT} 1 \mathrm{~b})$, carnitine palmitoyltransferase 2 (CPT2), and peroxisome proliferatoractivated receptor alpha (PPAR $\alpha$ ) in Epac2a-KO mice (Fig. 2C). Likewise, mRNA expression levels exhibited similar results to proteins (Fig. 2D). Remarkably, the expression of the UCP-1 protein in Epac2a-KO mice was higher than that in WT mice even at ambient temperature, and protein levels further increased during low-temperature exposure. After cold stress, we observed that the mRNA expression of cluster of differentiation 36 (Cd36), cytochrome c oxidase subunit VIIIb (Cox8b), and cytochrome complex (CytoC), which are associated with fatty acid uptake and mitochondrial oxidative metabolism, was higher in Epac2a-KO mice than in WT mice (Fig. 2E). To determine whether the hyperleptinemia observed in Epac2a-KO mice caused the increased cold endurance, leptin $(5 \mu \mathrm{g} / \mathrm{g}$ ) was administered intraperitoneally (i.p.) to WT mice, and then the mice were immediately exposed to a cold environment $\left(4^{\circ} \mathrm{C}\right)$ for $6 \mathrm{~h}$. Expectedly, the leptin-treated WT mice maintained a more stable body temperature than untreated WT mice (Fig. 2F). 


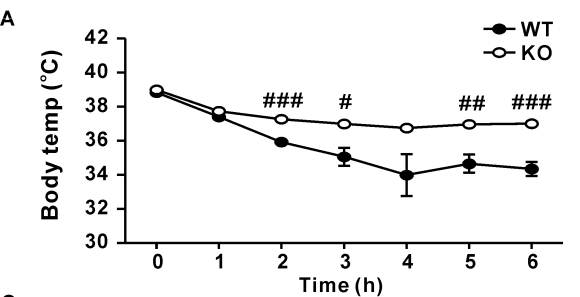

C
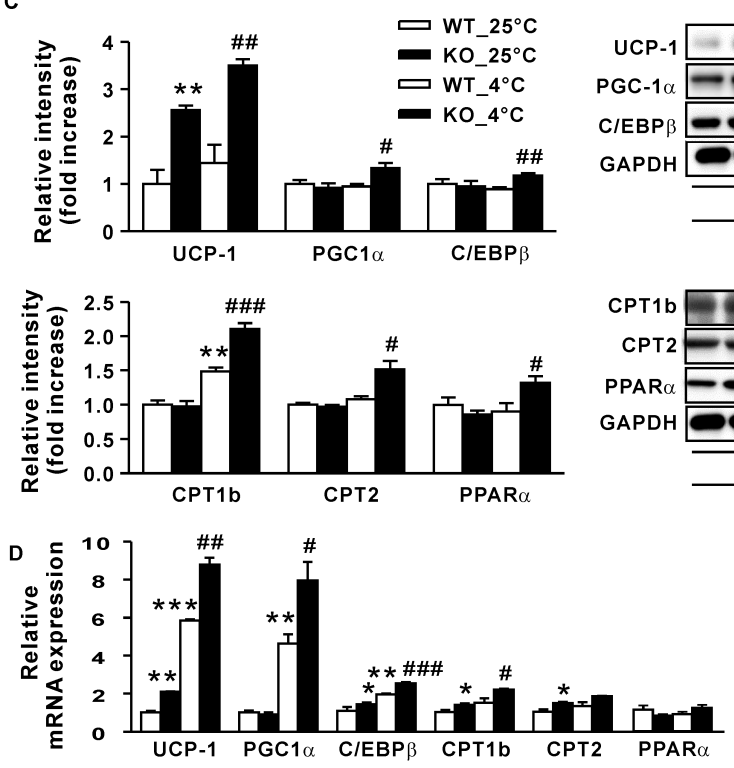

E
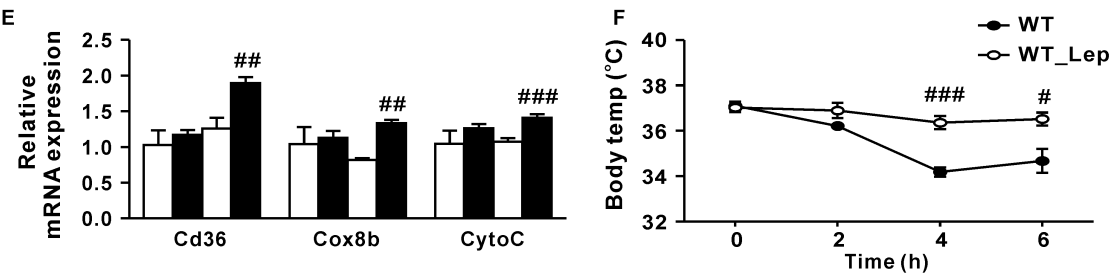
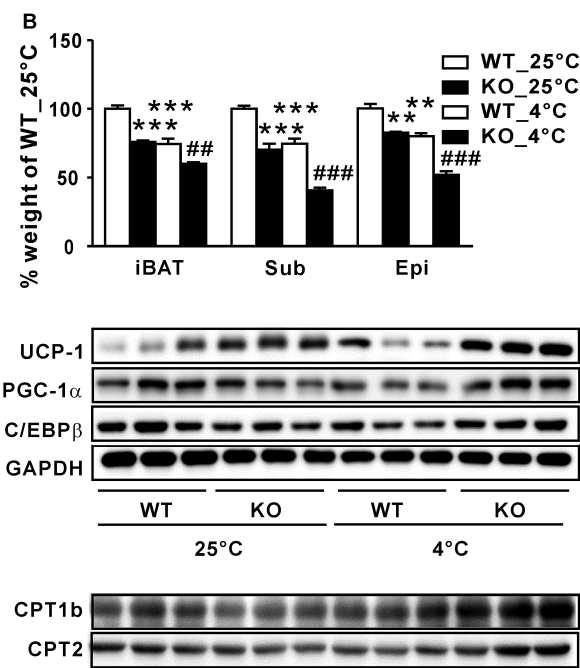

PPAR $\alpha-\infty-\infty-\infty-\infty$

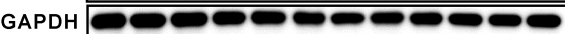

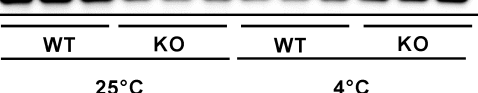

Fig. 2. Epac2a-KO mice display an enhanced response to the cold challenge. Changes in the body temperature $(A, n=9)$ and fat weight (B, $n=8)$ during a $6 \mathrm{~h}$ exposure to cold $\left(4^{\circ} \mathrm{C}\right)$. Protein and mRNA expression of thermogenic markers (C and D, $n=4$ ) and mRNA expression of fatty acid oxidation markers $(\mathrm{E}, n=8)$ in the iBAT of WT and KO mice. (F) Changes in the body temperature of WT mice with or without leptin administration during a 6 $\mathrm{h}$ exposure to cold $\left(4^{\circ} \mathrm{C}\right)$. WT mice in the leptin group were injected i.p. with leptin (5 $\mu \mathrm{g} / \mathrm{g}$ ) before cold exposure, whereas WT mice in the control group were injected i.p. with the same volume of PBS $(n=8)$. ${ }^{*} \mathrm{P}<0.05, * * \mathrm{P}<0.01$ and $* * * \mathrm{P}<0.001$ compared to WT_25 $5^{\circ}$. ${ }^{\#} \mathrm{P}<0.05,{ }^{\# \#} \mathrm{P}<0.01$, and \#\#\# $\mathrm{P}^{-}<0.001$ compared to WT $4^{\circ} \mathrm{C}$ without any treatment. Data represent the mean + SEM. Sub: subcutaneous fat; Epi: epididymal fat; Lep: leptin.

\section{Greater endurance of Epac2a-KO mice to dexamethasone-induced muscle atrophy}

The body weight of 7-week-old WT and Epac2a-KO mice did not differ (Fig. 3A). Dexamethasone treatment for 7 days (i.p.) significantly decreased the body weight of WT mice, but not that of Epac2a-KO mice. Accordingly, dexamethasone treatment reduced gastrocnemius muscle weight in WT mice, but not in Epac2a-KO mice. We then tested the functional performance of the gastrocnemius muscle with a treadmill exercise test. Treatment with dexamethasone significantly decreased the running time of the WT mice. Although dexamethasone treatment decreased the running time of Epac2a-KO mice, the decrease was less than that in WT mice (Fig. 3B). Consistent with the notion that dexamethasone damages skeletal muscle, serum creatine kinase levels in WT mice were elevated by dexamethasone treatment; however, the effect of dexamethasone in Epac2a-KO mice was minimal (Fig. 3C). Next, we evaluated the expression of proteins associated with protein synthesis and degradation in the gastrocnemius muscle with or without dexamethasone treatment (Fig. 3D). In WT mice, dexamethasone treatment significantly decreased the expression of p-AKT and p-mammalian target of rapamycin (p-mTOR), but increased the expression of p-eukaryotic initiation factor $4 \mathrm{E}$ (p-elF-4E), atrogin-1, and muscle RING-finger protein (MuRF), suggesting increased protein degradation and decreased protein synthesis. Conversely, in Epac2a-KO mice, dexamethasone treatment increased the protein expression of $\mathrm{p}$-AKT and p-mTOR, while the dexamethasone-mediated increase in p-elF-4E, atrogin-1, and MuRF expression observed in WT mice was attenuated. Dexamethasone-induced body weight reduction in WT mice shown in Fig. 3A was not due to the decrease in fat mass (Fig. 3E). To elucidate the increase in SNA and leptin signaling in 

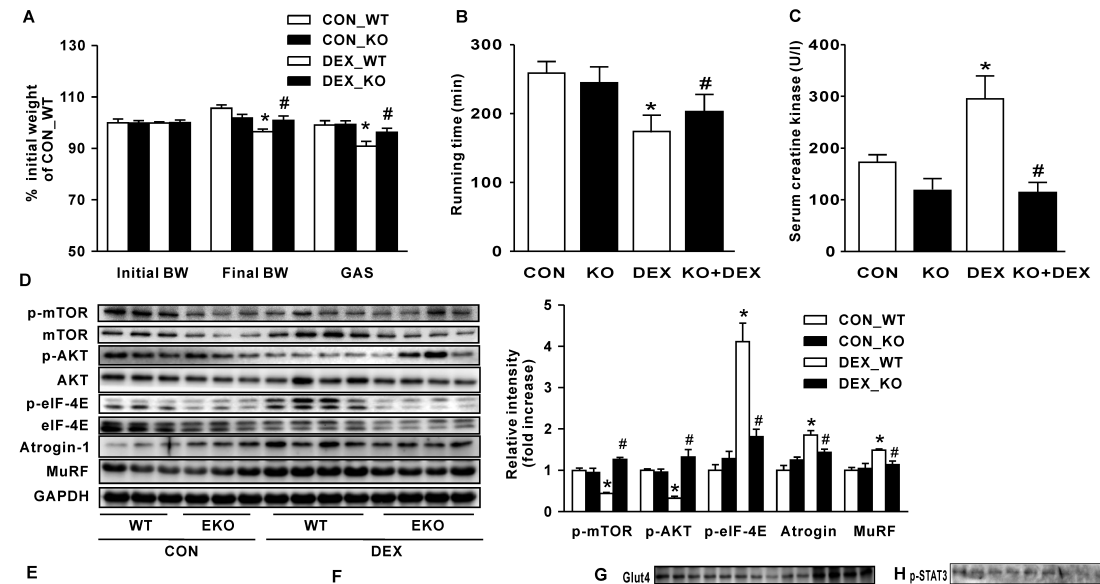

E
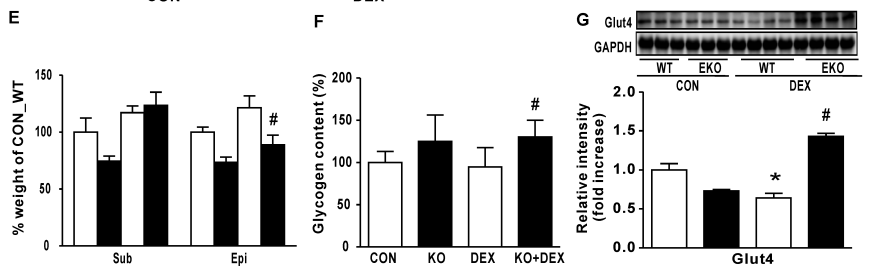

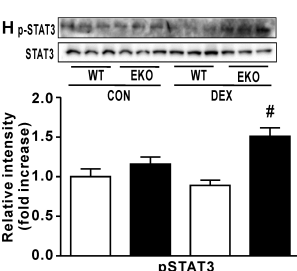

Fig. 3. Epac2a-KO mice better endure dexamethasone-induced muscle atrophy. (A) Changes in body weight and gastrocnemius muscle (GAS) weight following 7 days of dexamethasone (Dex) treatment (25 $\mathrm{mg} / \mathrm{kg} /$ day; $\mathrm{n}=15$ ). (B) Total running time on a treadmill exercise test without or with a 7-day dexamethasone treatment $(\mathrm{n}=11)$. (C) Serum creatine kinase activity without or with dexamethasone treatment. (D) The expression of proteins in the gastrocnemius muscle with or without dexamethasone treatment $(n=8)$. (E) Changes in fat weight following 7 days of dexamethasone treatment $(25 \mathrm{mg} / \mathrm{kg} /$ day; $\mathrm{n}=5)$. ( $\mathrm{F})$ Muscle glycogen content without or with dexamethasone treatment $(n=5)$. (G and $\mathrm{H}$ ) The expression of Glut4 and phosphorylation of Stat 3 in the gastrocnemius muscle with or without dexamethasone treatment $(n=4)$. ${ }^{*} \mathrm{P}<$ 0.05 compared to WT mice without dexamethasone treatment. ${ }^{\text {\# }} \mathrm{P}<0.05$ compared to WT mice with dexamethasone treatment. Data represent the mean \pm SEM. skeletal muscle, we measured glycogen content, glucose transporter type 4 (GLUT4) expression, and pSTAT3 expression from gastrocnemius muscle. Consistent with previous findings (18), glycogen content and protein level of GLUT4 were greater in $\mathrm{KO}$ mice treated with dexamethasone compared to WT mice treated with dexamethasone. Additionally, STAT3 phosphorylation was also higher in $\mathrm{KO}$ mice treated with dexamethasone, which is a direct signaling molecule for leptin. Furthermore, we observed more potentiated pPKA expression in $\mathrm{BBAT}$ and gastrocnemius muscle in Epac2a-KO mice (Supplementary Fig. 1). Cold stress also decreased triglyceride content in iBAT much more in $\mathrm{KO}$ mice than in WT mice (Supplementary Fig. 2).

\section{Effect of exogenous leptin treatment on dexamethasone-induced muscle atrophy}

WT mice were administered leptin (i.p.). After $45 \mathrm{~min}$, an ex vivo study of the gastrocnemius muscle was conducted. Following leptin treatment, the protein expression of $\mathrm{p}$-AKT and p-mTOR was increased, which indicates a potentiation of protein anabolism induced by exogenous leptin (Fig. 4A). In addition, chronic leptin treatment for 7 days ameliorated the dexamethasone-mediated decrease in the weight of the gastrocnemius muscle and the dexamethasone-mediated change in the protein expression of p-AKT and MuRF (Fig. 4B and 4C).

\section{DISCUSSION}

The present study demonstrates that leptin-mediated activation of SNA through the hypothalamus is not impaired in
Epac2a-KO mice. We confirmed that Epac2a expression was not detected in gastrocnemius muscle and BAT in WT mice (Supplementary Fig. 3). In addition, we demonstrated that skeletal muscle and BAT in Epac2a-KO mice have normal leptin and adrenergic signaling through their own respective plasmalemmal receptors. They are known to express both leptin and the leptin receptor, which is the long-form (ObRb) for typical intracellular leptin signaling through Janus kinase (JAK) and signal transducer and activator of transcription (STAT) (19).

In BAT, leptin was shown to increase glucose utilization through ObRb and the JAK/STAT pathway. It demonstrates that leptin increases the expression of some metabolic enzymes and PPAR activity in preadipocytes and BAT $(17,20)$. However, direct leptin action on UCP-1 expression in BAT was not observed, suggesting that thermogenesis in BAT is not directly associated with leptin receptor signaling. In fact, UCP-1 gene expression in BAT was induced by leptin, but through sympathetic innervation (21). Diet-induced obese mice, which are resistant to the anorectic effect of leptin but are hyperleptinemic, exhibited a higher iBAT temperature than mice fed a regular diet, whereas ob/ob mice that do not have the leptin gene showed a lower iBAT temperature (22). Interestingly, exogenous leptin administration in diet-induced obese mice and ob/ob mice increased iBAT temperature through the $\beta 3$ receptors, and leptin receptor blockade in the dorsomedial hypothalamus prevented the thermogenic effects of leptin, suggesting a leptin-mediated hypothalamus-SNA-BAT axis (23). Unresponsiveness to leptin for appetite control in obesity is observed in the pro-opiomelanocortin (POMC) 
A

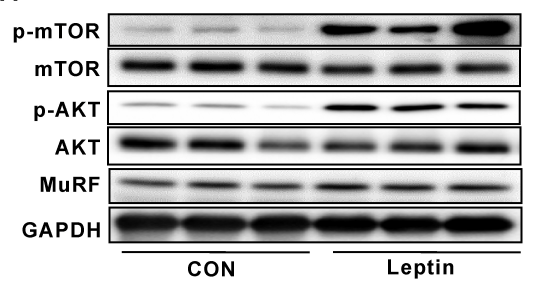

B

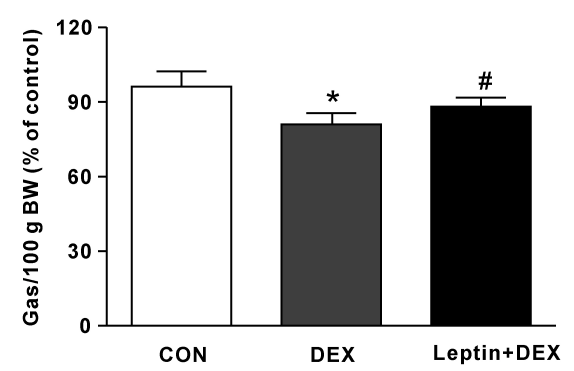

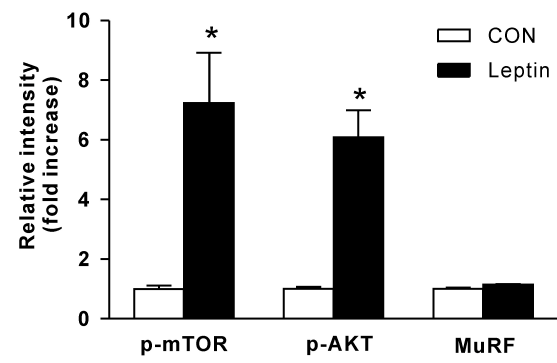

C

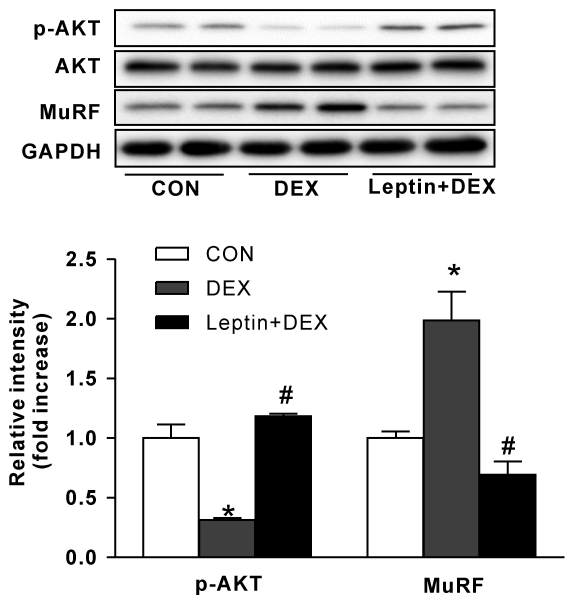

Fig. 4. Exogenous leptin induces muscle resistance to dexamethasone (Dex). (A) The expression of proteins in the gastrocnemius muscle of WT mice with or without leptin treatment. Leptin (5 $\mu \mathrm{g} / \mathrm{g})$ was administered i.p. $45 \mathrm{~min}$ before sample collection $(n=3)$. Changes in muscle weight $(B, n=8)$ and protein expression $(C, n=3)$ in the muscle after 7 days of Dex (25 $\mathrm{mg} / \mathrm{kg} /$ day) and/or leptin (5 $\mu \mathrm{g} / \mathrm{g} /$ day) treatment. $* \mathrm{P}<0.05$ compared to WT mice without any treatment. ${ }^{\#} \mathrm{P}<0.05$ compared to dexamethasone-treated WT mice. Data represent the mean \pm SEM neurons of ARH in MC4R-dependent manner. Leptin-mediated SNA for the increase in EE may occur via ARH (24). However, the dorsomedial hypothalamus is essential for transmitting the thermogenic signal bypassing $\mathrm{ARH}$ and exploiting MC4Rindependent pathway. Based on this, the present results suggest that endurance to cold stress in Epac2a-KO mice is primarily due to leptin signaling in the hypothalamic SNA, consistent with previous findings (4).

In skeletal muscle, leptin directly exerts its action, which is like the action of insulin, by facilitating glycogen synthesis and glucose uptake, and promoting cell survival. Leptin deficiency (e.g., ob/ob mice) caused decreased muscle mass (25-27) and increased expression of the muscle-wasting protein myostatin $(28,29)$, which was recovered by administration of exogenous leptin. Because the leptin receptor is expressed in human skeletal muscle, leptin may be an essential autocrine and paracrine muscle trophic factor (30). In addition, the sympathetic nervous system, mainly via $\beta 2$ receptors, increases muscle mass by activating the Akt/mTOR pathway (31-33), which has therapeutic potential against age-related muscle wasting, muscular dystrophy, and various muscle catabolic stresses (34). In Epac2a-KO mice, the skeletal muscle is more anabolic in nature, and it can endure when treatment is prolonged with the glucocorticoid, dexamethasone. Therefore, it may be plausible that augmented leptin signaling in Epac2a-KO mice fortifies the skeletal muscle through its own leptin receptors, as well as its own adrenergic receptors via hypothalamic leptin signaling.

In conclusion, like arterial blood pressure, which is elevated in diet-induced obesity, hypothalamic leptin resistance in the absence of Epac2a cannot block leptin signaling to enhance sympathetic outflow. This suggests that Epac2a in the hypothalamus only affects leptin-mediated signaling for appetite control (22). The present results also suggest that leptin receptor-induced signaling in skeletal muscle and BAT might be normal in Epac2a-KO mice, unlike the leptin receptor signaling in the hypothalamus.

\section{MATERIALS AND METHODS}

Detailed information is provided in the Supplementary Material.

\section{ACKNOWLEDGEMENTS}

This study was supported by a grant from the National Research Foundation of Korea (NRF) Grant funded by the Korean Government (MSIP) (No. 2014R1A5A2010008 and NRF-2013R1A2A2A01068220). 


\section{CONFLICTS OF INTEREST}

The authors have no conflicting interests.

\section{REFERENCES}

1. Seino S and Shibasaki T (2005) PKA-dependent and pka-independent pathways for cAMP-regulated exocytosis. Physiol Rev 85, 1303-1342

2. Takahashi H, Shibasaki T, Park JH et al (2015) Role of Epac2A/Rap1 signaling in interplay between incretin and sulfonylurea in insulin secretion. Diabetes 64, 1262-1272

3. Schmidt M, Dekker FJ and Maarsingh H (2013) Exchange protein directly activated by cAMP (epac): a multidomain CAMP mediator in the regulation of diverse biological functions. Pharmacol Rev 65, 670-709

4. Hwang M, Go Y, Park JH et al (2017) Epac2a-null mice exhibit obesity-prone nature more susceptible to leptin resistance. Int J Obes 41, 279-288

5. Huxing C, Miguel L and Kamal R (2017) The cellular and molecular bases of leptin and ghrelin resistance in obesity. Nat Rev Endocrinol 13, 338-351

6. Morgan DA, Thedens DR, Weiss $R$ and Rahmouni $K$ (2008) Mechanisms mediating renal sympathetic activation to leptin in obesity. Am J Physiol Regul Integr Comp Physiol 295, R1730-R1736

7. Caro JF, Kolaczynski JW, Nyce MR et al (1996) Decreased cerebrospinal-fluid/serum leptin ratio in obesity: a possible mechanism for leptin resistance. Lancet 348, 159-161

8. Banks WA (2001) Leptin transport across the blood-brain barrier: implications for the cause and treatment of obesity. Curr Pharm Des 7, 125-133

9. Magni P, Liuzzi A, Ruscica M et al (2005) Free and bound plasma leptin in normal weight and obese men and women: relationship with body composition, resting energy expenditure, insulin sensitivity, lipid profile and macronutrient preference. Clin Endocrinol 62, 189-196

10. Houseknecht KL, Mantzoros CS, Kuliawat R, Hadro E, Flier JS and Kahn BB (1996) Evidence for leptin binding to proteins in serum of rodents and humans: modulation with obesity. Diabetes 45, 1638-1643

11. Schwartz MW, Peskind E, Raskind M, Boyko EJ and Porte D Jr (1996) Cerebrospinal fluid leptin levels: relationship to plasma levels and to adiposity in humans. Nat Med 2, 589-593

12. Nickki O, Parinaz M, Belen R et al (2015) Diet-induced obese mice retain endogenous leptin action. Cell Metab $6,877-882$

13. Haynes WG, Morgann DA, Djalali A et al (1999) Interactions between the melanocortin system and leptin in control of sympathetic nerve traffic. Hypertension 33, 542-547

14. Cone RD (2005) Anatomy and regulation of the central melanocortin system. Nat Neurosci 8, 571-578

15. Cowley MA, Smart JL, Rubinstein M et al (2001) Leptin activates anorexingenic POMC neurons through a neural network in the arcuate nucleus. Nature 411, 480-484

16. Simonds SE, Pryor JT, Ravussin E et al (2014) Leptin mediates the increase in blood pressure associated with obesity. Cell 159, 1404-1416

17. Siegrist-Kaiser CA, Pauli V, Juge-Aubry CE et al (1997) Direct effects of leptin on brown and white adipose tissue. J Clin Invest 100, 2858-2864

18. Maroni P, Bendinelli R, Piccoletti R (2003) Early intracellular events induced by in vivo leptin treatment in mouse skeletal muscle. Mol Cell Endocrinol 201, 109-121

19. Gema F (2006) Intracellular signalling pathways activated by leptin. Biochem J 393, 7-20

20. Bai Y, Zhang SY, Kim KS, Lee JK and Kim KH (1996) Obese gene expression alters the ability of 30A5 preadipocytes to respond to lipogenic hormones. J Biol Chem 271, 13939-13942

21. Bartness TJ, Vaughan CH and CK Song (2010) Sympathetic and sensory innervation of brown adipose tissue. Int J Obes (Lond) 34, S36-S42

22. Enriori PJ, Sinnayah P, Simonds SE, Rudaz CG and Cowley MA (2011) Leptin action in the dorsomedial hypothalamus increases sympathetic tone to brown adipose tissue in spite of systemic leptin resistance. J Neurosci 31, 12189-12197

23. Hwa JJ, Fawzi AB, Graziano MP et al (1997) Leptin increase energy expenditure and selectively promotes fat metabolism in ob/ob mice. Am J Physiol 272, R1204-1209

24. Scott MM, Lachey JL, Sternson SM et al (2009) Leptin targets in the mouse brain. J Comp Neurol 514, 518-532

25. Margetic S, Gazzola C, Pegg GG and Hill RA (2002) Leptin: a review of its peripheral actions and interactions. Int J Obes Rel Metab Disord 26, 1407-1433

26. Sainz N, Rodriguez A, Catalan V, Becerril S and Ramirez B (2009) Leptin administration favors muscle mass accretion by decreasing fox03a and Increasing PGC-1a in ob/ob mice. PLoS One 4, e6808

27. Hamrick MW, Pennington C, Newton D, Xie D and Isales CM (2004) Leptin deficiency produces contrasting phenotypes in bones of the limb and spine. Bone 34, 376-383

28. Pansters NA, Langen RC, Wouters E and Schols AM (2012) Synergistic stimulation of myogenesis by glucocorticoid and IGF-I signaling. J Appl Phys 114, 1329-1339

29. Allen D, Clearly A, Speaker K et al (2008) Myostatin, activin receptor IIB, and follistatin-like-3 gene are altered in adipose tissue and skeletal muscle of obese mice. Am J Physiol Endocrinol Metab 294, E918-E927

30. Hamrick MW, Herberg S, Arounleut P et al (2010) The adipokine leptin increases skeletal muscle mass and significantly alters skeletal muscle miRNA expression profile in aged mice. Biochem Biophys Res Commun 400, 379-383

31. Lynch GS and Ryall JG (2008) Role of $\beta$-adrenoceptor signaling in skeletal muscle: implication for muscle wasting and disease. Physiol Rev 88, 729-767

32. Maroni P, Bendinelli P and Piccoletti R (2003) Early Intracellular events induced by in vivo leptin treatment in mouse skeletal muscle. Mol Cell Endocrinol 201, 109-121

33. Hasselgren PO, Wray C and Mammen J (2002) Molecular regulation of muscle cachexia: it may be more than the proteasome. Biochem Biophys Res Commun 290, 1-10

34. Romanick M, Thompson LV and Brown-Borg HM (2013) Murine models of atrophy, cachexia, and sarcopenia in skeletal muscle. Biochim Biophys Acta 1832, 1410-1420 